\title{
Dimension Reduction in Multivariate Linear Regression
}

\author{
William W.S. Chen \\ Department of Statistics, the George Washington University \\ Washington D.C. 20013 \\ williamwschen@gmail.com
}

\begin{abstract}
We defined some elementary terminology. It includes the vector space, linear combination, set of independent vectors, dependent vectors, basis of vector space, and direct sum of subspaces. This theory can help us lower the dimension of a given vector spaces. We apply to multivariate linear multiple regression analysis. It not only simplifies the computation and eases the interpretation, but also reduce the rate of errors. Cook (2010) developed an envelope model for the same reason. The main objective in that model is decomposing the covariance matrix into the sum of two matrices, each of whose column spaces either contains, or is orthogonal to, the subspace containing the mean. In other words, break the covariance matrix into the direct sum of the subspaces.

Mathematical Subject Classification: 15-00

Keywords and Phrases: basis of the vector space, direct sum of subspaces, decompose the covariance matrix, envelope model, finite dimensional vector space, linear combinations of vector, linearly dependent vectors, linearly independent vectors, multivariate linear regression, span of the vector space.

\section{Introduction}

Author worked for Internal Revenue Service for a long period of time. That organization has over onehundred thousand variables available for study. It is not uncommon to study more than a hundred variables simultaneously at only one time. For example, in a case study there are 130 variables needed to find a relationship between the variables and predict its future trend. We need to run a multivariate regression analysis. Our experiences are calling a conference meeting with the subject matter group members. Using their suggestions, we can break down the variables into smaller groups. Let say 130 variables are cut into six groups of 20 variables each, and 10 variables in the remainder one. In this way, it is not only easier to run the program, but it will also help to interpret the output, and reduce the chance to make errors. Cook (2010) developed the envelope model that decomposed the covariance matrix into the direct sum of two matrices, each of whose column spaces either contains or is orthogonal to the subspace containing the mean. The objective of his model is consistent with ours.
\end{abstract}




\section{Linear Transformation}

Let $\mathrm{V}$ be $\mathrm{n}$-dimensional vector space over a field $\mathrm{F}$ and let $v_{1}, \ldots v_{N}$ be a basis of $\mathrm{V}$ over $\mathrm{F}$. If $T \in A(\mathrm{~V})$, then $\mathrm{T}$ is determined on any vector as soon as we know its action on a basis of $\mathrm{V}$. Since $\mathrm{T}$ maps $\mathrm{V}$ into $\mathrm{V}$, $\mathrm{V}_{1} T, v_{2} T, \ldots . v_{n} T$ must all be in $\mathrm{V}$. As elements of $\mathrm{V}$, each of these is a realization in a unique way as a linear combination of $v_{1}, \ldots v_{N}$ over $F$. Thus,

$$
\begin{aligned}
& \mathrm{v}_{1} T=\alpha_{11} v_{1}+\alpha_{12} v_{2}+\ldots \ldots \ldots+\alpha_{1 n} v_{n} \\
& \mathrm{v}_{2} T=\alpha_{21} v_{1}+\alpha_{22} v_{2}+\ldots \ldots \ldots+\alpha_{2 n} v_{n} \\
& \mathrm{v}_{\mathrm{n}} T=\alpha_{n 1} v_{1}+\alpha_{n 2} v_{2}+\ldots \ldots \ldots+\alpha_{n n} v_{n}
\end{aligned}
$$

Where each $\alpha_{\mathrm{ij}} \in F$ This system of equation can be written more compactly as

$$
\mathrm{v}_{\mathrm{i}} T=\sum_{j=1}^{n} \alpha_{i j} v_{j} \quad \mathrm{i}=1,2 \ldots . . \mathrm{n} \mathrm{j}
$$

The ordered set of $\mathrm{n}^{2}$ numbers $\alpha_{\mathrm{ij}}$ in F completely describes T. They will serve as the measure of representing $\mathrm{T}$.

Definition 2.1

Let $\mathrm{V}$ be an $\mathrm{n}$ dimensional vector space over $\mathrm{F}$ and let $v_{1}, \ldots . v_{n}$

be a basis for v over $\mathrm{F}$. If $\mathrm{T} \in \mathrm{A}(\mathrm{V})$ then the matrix of $\mathrm{T}$ in the basis $v_{1}, \ldots . v_{n}$ written as $\mathrm{m}(\mathrm{T})$, is

$$
\begin{gathered}
m(T)=\left(\begin{array}{ccccc}
\alpha_{11} & \alpha_{12} & \cdot & \cdot & \alpha_{1 n} \\
\alpha_{21} & \alpha_{22} & \cdot & \cdot & \alpha_{2 n} \\
\cdot & \cdot & \cdot & \cdot & \cdot \\
\cdot & \cdot & \cdot & \cdot & \cdot \\
\alpha_{n 1} & \alpha_{n 2} & \cdot & \cdot & \alpha_{n n}
\end{array}\right) \\
\text { where } \mathrm{v}_{\mathrm{i}} T=\sum_{j=1}^{n} \alpha_{i j} v_{j}
\end{gathered}
$$

Example 2.1 Let the vector space $\mathrm{V}$ of $2 \times 2$ matrix over $\mathrm{R}$ and the following usual basis $\mathrm{E}$ of $\mathrm{V}$.

$$
\mathrm{E}=\left\{\mathrm{E}_{1}=\left(\begin{array}{ll}
1 & 0 \\
0 & 0
\end{array}\right), \mathrm{E}_{2}=\left(\begin{array}{ll}
0 & 1 \\
0 & 0
\end{array}\right), \mathrm{E}_{3}=\left(\begin{array}{ll}
0 & 0 \\
1 & 0
\end{array}\right), \mathrm{E}_{4}=\left(\begin{array}{ll}
0 & 0 \\
0 & 1
\end{array}\right)\right\}
$$


Let $M=\left(\begin{array}{ll}1 & 2 \\ 3 & 4\end{array}\right)$ and $\mathrm{T}_{1}$ be the linear transformation on $\mathrm{V}$ defined by $\mathrm{T}_{1}(E)=M E$. Find the matrix representation of $T_{1}$ relative to the above usual basis of $\mathrm{V}$.

$$
\begin{aligned}
& T_{1}\left(E_{1}\right)=M E_{1}=\left(\begin{array}{ll}
1 & 2 \\
3 & 4
\end{array}\right)\left(\begin{array}{ll}
1 & 0 \\
0 & 0
\end{array}\right)=\left(\begin{array}{ll}
1 & 0 \\
3 & 0
\end{array}\right)=1 E_{1}+0 E_{2}+3 E_{3}+0 E_{4} \\
& T_{1}\left(E_{2}\right)=M E_{2}=\left(\begin{array}{ll}
1 & 2 \\
3 & 4
\end{array}\right)\left(\begin{array}{ll}
0 & 1 \\
0 & 0
\end{array}\right)=\left(\begin{array}{ll}
0 & 1 \\
0 & 3
\end{array}\right)=0 E_{1}+1 E_{2}+0 E_{3}+3 E_{4} \\
& T_{1}\left(E_{3}\right)=M E_{3}=\left(\begin{array}{ll}
1 & 2 \\
3 & 4
\end{array}\right)\left(\begin{array}{ll}
0 & 0 \\
1 & 0
\end{array}\right)=\left(\begin{array}{ll}
2 & 0 \\
4 & 0
\end{array}\right)=2 E_{1}+0 E_{2}+4 E_{3}+0 E_{4} \\
& T_{1}\left(E_{4}\right)=M E_{4}=\left(\begin{array}{ll}
1 & 2 \\
3 & 4
\end{array}\right)\left(\begin{array}{ll}
0 & 0 \\
0 & 1
\end{array}\right)=\left(\begin{array}{ll}
0 & 2 \\
0 & 4
\end{array}\right)=0 E_{1}+2 E_{2}+0 E_{3}+4 E_{4}
\end{aligned}
$$

Since $\operatorname{dim} V=4$, any matrix representation of a linear transformation on $V$ must be a 4-square matrix.

Hence $\left|T_{1}\right|_{E}=\left(\begin{array}{cccc}1 & 0 & 2 & 0 \\ 0 & 1 & 0 & 2 \\ 3 & 0 & 4 & 0 \\ 0 & 3 & 0 & 4\end{array}\right)$

Example 2.2 Let $M=\left(\begin{array}{ll}a & b \\ c & d\end{array}\right)$ and $\mathrm{T}_{2} \mathrm{~V}->\mathrm{V}$ be the lineartransformation on $\mathrm{V}$ defined by $\mathrm{T}_{2}(E)=E M$. Find the matrix representation of $\mathrm{T}_{2}$ in the above usual basis of $\mathrm{V}$.

$$
\begin{aligned}
& T_{2}\left(E_{1}\right)=E_{1} M=\left(\begin{array}{ll}
1 & 0 \\
0 & 0
\end{array}\right)\left(\begin{array}{ll}
a & b \\
c & d
\end{array}\right)=\left(\begin{array}{ll}
a & b \\
0 & 0
\end{array}\right)=a E_{1}+b E_{2}+0 E_{3}+0 E_{4} \\
& T_{2}\left(E_{2}\right)=E_{2} M=\left(\begin{array}{ll}
0 & 1 \\
0 & 0
\end{array}\right)\left(\begin{array}{ll}
a & b \\
c & d
\end{array}\right)=\left(\begin{array}{ll}
c & d \\
0 & 0
\end{array}\right)=c E_{1}+d E_{2}+0 E_{3}+0 E_{4} \\
& T_{2}\left(E_{3}\right)=E_{3} M=\left(\begin{array}{ll}
0 & 0 \\
1 & 0
\end{array}\right)\left(\begin{array}{ll}
a & b \\
c & d
\end{array}\right)=\left(\begin{array}{ll}
0 & 0 \\
a & b
\end{array}\right)=0 E_{1}+0 E_{2}+a E_{3}+b E_{4} \\
& T_{2}\left(E_{4}\right)=E_{4} M=\left(\begin{array}{ll}
0 & 0 \\
0 & 1
\end{array}\right)\left(\begin{array}{ll}
a & b \\
c & d
\end{array}\right)=\left(\begin{array}{ll}
0 & 0 \\
c & d
\end{array}\right)=0 E_{1}+0 E_{2}+c E_{3}+d E_{4}
\end{aligned}
$$




$$
\text { Hence }\left|T_{2}\right|_{E}=\left(\begin{array}{cccc}
a & b & 0 & 0 \\
c & d & 0 & 0 \\
0 & 0 & a & b \\
0 & 0 & c & d
\end{array}\right)=\left(\begin{array}{cc}
M & 0 \\
0 & M
\end{array}\right)
$$

\section{Define Terms}

In this section we will define a sequence of useful terminology.

It includes vector space, subspace of a vector space, linear combination of the vector, span of the vectors, set of linear independent vectors, basis of the vector space, dimension of vector space. All these terms are required for us to understand dimension reduction. Define the vector space as follow.

Definition 3.1: A nonempty set $\mathrm{V}$ is said to be a vector space over a field $\mathrm{F}$ if $\mathrm{V}$ is an abelian group under an operation which we denote by +, and if for every $\alpha \in F, v \in V$ there is defined an element written as $\alpha V \in V$ subject to:

$$
\text { (1) } \alpha(v+w)=\alpha v+\alpha w,(2)(\alpha+\beta) v=\alpha v+\beta v,(3) \alpha(\beta \mathrm{v})=(\alpha \beta) \mathrm{v},
$$

(4) $1 \mathrm{v}=\mathrm{v}$ for all $\alpha, \beta \in \mathrm{F}, \mathrm{v}, \mathrm{w} \in \mathrm{V}$.

Where 1 represents the unit element of $\mathrm{F}$ under multiplication.

definition 3.2: If $\mathrm{V}$ is a vector space over $\mathrm{F}$ if $W \subset V$ then $\mathrm{W}$ is a subspace of $\mathrm{V}$ if under the operation of $\mathrm{V}, \mathrm{W}$, itself, forms

a vector space over $\mathrm{F}$. Equivalently, $\mathrm{W}$ is a subspace of $\mathrm{V}$ whenever $w_{1}, w_{2} \in W, \alpha \beta \in \mathrm{F}$ implies that $\alpha w_{1}+\beta w_{2} \in W$.

Definition 3.3: A linear combination of the vector space

is a sum of scalar multiples of these vectors, that is,

$c_{1} v_{1}+c_{2} v_{2}+, \ldots . c_{n} v_{n}$, for some scalar coefficients, $c_{1}, c_{2}, \ldots . c_{n} \in F$. If $\mathrm{S}$ is a set of vectors in $\mathrm{V}$, a linear combination of vectors in $S$ is a vector of the form $c_{1} v_{1}+c_{2} v_{2}+, \ldots . c_{n} v_{n}$ with $\mathrm{i} \in \mathrm{N}, \mathrm{v}_{\mathrm{i}} \in S, \mathrm{c}_{\mathrm{i}} \in F$

Definition 3.4: The span of the vectors $C_{1}, C_{2}, \ldots . c_{n} \in V$ is the set of all linear combinations of these vectors, denoted by $\operatorname{span}\left(C_{1}, C_{2}, \ldots . C_{n}\right)$. If $\mathrm{S}$ is a (finite or infinite) set of vectors in $\mathrm{V}$, then the span of $S$, denoted by $\operatorname{span}(S)$, is the set of all linear combinations of vectors in $S$. If $V=\operatorname{span}(S)$, then $S$ spans the vector space $\mathrm{V}$.

Definition 3.5: A (finite or infinite) set of vectors $\mathrm{S}$ in $\mathrm{V}$ is linearly independent if the only linear combination of distinct vectors in $\mathrm{S}$ that produces the zero vector is a trivial linear combination. That is, if $\mathrm{V}_{\mathrm{i}}$ are distinct vectors in $\mathrm{S}$ and $c_{1} V_{1}+c_{2} V_{2}+, \ldots . c_{n} v_{n}=0$, then $\mathrm{C}_{1}=\mathrm{C}_{2}=\ldots .=\mathrm{C}_{\mathrm{n}}=0$. Vectors that are not 
William W.S. Chen; Dimension Reduction in Multivariate Linear Regression. Transactions on Machine Learning and Artificial Intelligence, Volume 7 No 1 February (2019); pp: 42-49

linearly independent are linear dependent. That is, there exist distinct vectors $v_{1}, v_{2}, \ldots . v_{n} \in S$ and $c_{1}, c_{2}, \ldots . c_{n}$ not all 0 such that

$c_{1} v_{1}+c_{2} v_{2}+, \ldots . c_{n} v_{n}=0$.

Definition 3.6: Let $V$ be a vector space over a field $F$. A set of vectors $B$ in a vector space $V$ is a basis for $V$ if (1) B is a linearly independent set and (2) Span(B)=V.

Definition 3.7: The number of vectors in a basis for a vector space $V$ is the dimension of $V$ denoted by $\operatorname{dim}(\mathrm{V})$.

Definition 3.8: The sum of two subspaces is direct if and only if the intersection of these subspaces is zero.

Since our objective is reduction the dimension of multivariate regression vector space using base theory. The following theorems can be found in $\mathrm{Mal}{ }^{\wedge} \mathrm{Cev}(1963)$ linear algebra. We only claim the theorems and not repeat its proof.

Theorem 3.1 The dimension of the sum of two linear subspaces is the sum of their dimensions minus the dimension of their intersection.

Proof: see $\mathrm{Mal}{ }^{\wedge} \mathrm{Cev}(1963)$, page 50. Alternatively, see Cook,R.D.,

Li,B. and Chiaromonte,F.(2010), page 936, Corollary 3.1.

Theorem 3.2 The dimension of a direct sum of subspaces is the sum of their dimension.

Proof: By theorem 3.1 the dimension of the sum is the sum of the dimensions minus the dimension of the intersection. From the definition 3.8, the intersection of the subspaces is zero

and has dimension zero. Therefore, the dimension of the direct sum of two subspaces is the sum of their dimensions.

If the number of summands is greater than 2, the proof carried out by induction. From theorem 3.2 we have the following.

Theorem 3.3 If a subspace $A$ is the direct sum of subspaces

$A_{1}, \ldots \ldots A_{n}$, then taking a basis $a_{i 1}^{\prime} \ldots . . a_{i m_{i}}^{\prime}$ of each subspace $A_{i}$

for $i=1, \ldots . n$ and combining these bases into one system,

$a_{11} \ldots . . a_{1 m_{1}} \ldots \ldots a_{s 1} \ldots . . a_{s m_{1}}$ we obtain a Basis of the subspace $\mathrm{A}$.

The above definitions and three theorems are the foundation of our applications. More of the related facts will be discussed in the section 4 . We now give some examples to demonstrate its the real meaning and its possible applications.

Example 3.1 If we are given that 


$$
\begin{aligned}
& A_{1}=\left(\begin{array}{ll}
2 & 3 \\
1 & 5
\end{array}\right), A_{2}=(1 \quad 2), A_{3}=(3) \text { then } \\
& A_{1} \oplus A_{2} \oplus A_{3}=\left(\begin{array}{lllll}
2 & 3 & 0 & 0 & 0 \\
1 & 5 & 0 & 0 & 0 \\
0 & 0 & 1 & 2 & 0 \\
0 & 0 & 0 & 0 & 3
\end{array}\right)
\end{aligned}
$$

Example 3.2 Let $\mathbf{B}=\left\{V_{1} \ldots \ldots V_{n}\right\}$ be a basis for $V$ then

$$
V=\operatorname{span}\left(V_{1}\right) \oplus \ldots \ldots \oplus \operatorname{span}\left(V_{n}\right)
$$

Example 3.3 In the direct sum $R(x ; 2) \times R^{2 \times 2}$

$$
\left(2 x^{2}+7,\left(\begin{array}{ll}
1 & 2 \\
3 & 4
\end{array}\right)\right)+3\left(x^{2}+4 x-2,\left(\begin{array}{cc}
0 & 1 \\
-1 & 0
\end{array}\right)\right)=\left(5 x^{2}+12 x+1,\left(\begin{array}{ll}
1 & 5 \\
0 & 4
\end{array}\right)\right)
$$

\section{Some Facts}

Although in section 3 we have defined the terms of basis and dimension of a vector space, it may not be good enough in actual application. We realize that these two terms are critical and useful to us. We list more facts to help us to more deeply understand these two terms. Fact 1: Every vector space has a basis. Fact 2: The standard basis for $F^{n}$ is a basis for $F^{n}$, and so $\operatorname{dim} F^{n}=n$. Fact 3: A basis B in a vector space $V$ is the largest set of linearly independent vectors in $V$ that contain $B$, and it is the smallest set of vectors in $V$ that contains $B$ and spans $V$. Fact 4 : The empty set is a basis for the trivial vector space $\langle 0\rangle$, and $\operatorname{dim}\langle 0\rangle=0$. Fact 5: If $S$ is a linearly independent set in a vector space $V$, then $S$ can be expanded, if necessary, to a basis for $\mathrm{V}$. Fact 6 : If the set $S=\left\langle v_{1}, \ldots . v_{p}>\right.$ spans a vector space $\mathrm{V}$, then some subset of $\mathrm{S}$ forms a basis for $\mathrm{V}$. For example, if one of the vectors, say $v_{i}$, is a linear combination of the remaining vectors, then the set formed from $\mathrm{S}$ by removing $v_{i}$, will be "closer" to a basis for $\mathrm{V}$. This process can be continued until the remaining vectors form a basis for $\mathrm{V}$.

Fact 7: If a vector space $V$ has a basis containing $n$ vectors, then every basis of $V$ must contain $n$ vectors. Similarly if $\mathrm{V}$ has infinite basis, then every basis of $\mathrm{V}$ must be infinite. So the dimension of $\mathrm{V}$ is unique. Fact 8: Let $\operatorname{dim}(V)=n$ and let $S$ be a set containing $n$ vectors. The following are equivalent: (8.1) $S$ is a basis for V. (8.2) S span V. (8.3) $\mathrm{S}$ is linear independent. Fact 9 : If $\operatorname{dim}(\mathrm{V})=\mathrm{n}$, then any subset of $\mathrm{V}$ containing more than $\mathrm{n}$ vectors is linearly dependent. Fact 10 : If $B=<b_{1}, \ldots . . b_{p}>$ is a basis for a vector space $\mathrm{V}$, then each $x \in V$ can be expressed as a unique linear combination of the vectors in $\mathrm{B}$. That is, for each $x \in V$ there is a unique set of scalars $c_{1}, c_{2}, \ldots . . c_{p}$ such that $x=c_{1} b_{1}+c_{2} b_{2}+\ldots \ldots+c_{p} b_{p}$. The concept of direct sum is the critical important to us. In section 3 we have clearly defined the direct sum and three important theorems. We found that the following fact can help us in real applications. Fact 1 : $W=W_{1} \oplus W_{2}$ if and only if $W=W_{1}+W_{2}$ and $W_{1} \cap W_{2}=<0>$ Fact 2: If $W$ is a subspace of $\mathrm{V}$, 
then there exists a subspace $\mathrm{U}$ of $\mathrm{V}$ such that $\mathrm{V}=\mathrm{W} \oplus \mathrm{U}$. Note that $\mathrm{U}$ is not usually unique. Fact 3 : Let $\mathrm{W}=\mathrm{W}_{1}+\ldots .+\mathrm{W}_{\mathrm{n}}$. The following statements are equivalent: (3.1) Let $\mathrm{W}=\mathrm{W}_{1} \oplus \ldots \oplus \mathrm{W}_{\mathrm{n}}$. That is, for all $i=1 \ldots n$,

we have $\mathrm{W}_{\mathrm{i}} \cap \sum_{j \neq i} W_{j}=<0>$. (3.2) $\mathrm{W}_{\mathrm{i}} \cap \sum_{j=1}^{i-1} W_{j}=<0>$ for all $\mathrm{i}=2, \ldots \ldots . \mathrm{n}$

(3.3) For each $\mathrm{W} \in \mathrm{W}, \mathrm{w}$ can be expressed in exactly one way as a sum of vectors in $\mathrm{W}_{1} \ldots \mathrm{W}_{\mathrm{n}}$. That is, there exists a unique $\mathrm{W}_{\mathrm{i}} \in \mathrm{W}_{\mathrm{i}}$, such that $\mathrm{W}=\mathrm{W}_{1}+\ldots . .+\mathrm{W}_{\mathrm{n}}$. (3.4) The subspace $w_{i}$, for $i=1 \ldots . n$ are independent. (3.5) If $B_{i}$ is an ordered basis for $W_{i}$, then $B=\bigcup_{i=1}^{n} B_{i}$ is an ordered basis for $W$. Fact 4 : If $B$ is a basis for $V$ and $B$ is partitioned into disjoint subset $B_{i}$, for $i=1 \ldots \ldots . n$. then $V=\operatorname{span}\left(B_{1}\right) \oplus \ldots \ldots . . \oplus \operatorname{span}\left(B_{n}\right)$. Fact 5 : If $S$ is a linearly independent subset of $V$ and $S$ is partitioned into disjoint subsets $S_{i}$, for $i=1 \ldots n$, then the subspaces $\operatorname{span}\left(\mathrm{S}_{1}\right), \ldots \ldots . . \operatorname{span}\left(\mathrm{S}_{\mathrm{n}}\right)$ are independent. Fact 6 : If $\mathrm{V}$ is finite dimensional and $\mathrm{V}=\mathrm{W}_{1}+\ldots \ldots . .+\mathrm{W}_{\mathrm{n}}$, then $\operatorname{dim}(\mathrm{V})=\operatorname{dim}\left(\mathrm{W}_{1}\right)+\ldots \ldots .+\operatorname{dim}\left(\mathrm{W}_{\mathrm{n}}\right)$ if and only if $\mathrm{V}=\mathrm{W}_{1} \oplus \ldots \ldots . \oplus \mathrm{W}_{\mathrm{n}}$.

\section{Concluding Remarks}

The Multivariate general linear multiple regression model is given by $Y=X B+E$ where $Y$ is $n \times p, X$ is $n \times q, B$ is qxp, and $E$ is nxp error matrix. If we move $E$ matrix to the other side of equation and combine with $Y$ matrix, then we can view $X$ matrix as a linear transformation on the parameter matrix $B$ to the response matrix $Y$. This paper is seeking some square submatrix $W_{i}$ such that $W_{i} \cap \sum_{j \neq i} W_{j}=<0>W_{i}$ for $\mathrm{i}=1,2, \ldots \mathrm{p}$ and $\mathrm{W}=\mathrm{W}_{1} \oplus \ldots . . \oplus \mathrm{W}_{\mathrm{p}}$. We are sure that such square matrix exist as can be seen from example 2.2. However, the method to identify such submatrix from the given data set may not be trivial. Compare to the Cook (2010) envelope model, we found that our basic objective to reduce the dimension of vector space is identical. Envelope model attempt to decompose the covariance matrix into the direct sum of two submatrix, each of whose column space either contain or is orthogonal to the subspace containing the mean. The only way to do this is to create a split based on the eigenvector of the covariance. Of course, this will lead to a large computer related computation.

\section{REFERENCES}

[1] Anderson, T.W. (1958). An Introduction to Multivariate Statistical Analysis. Second Edition, Wiley, New York.

[2] Cook, R.D. (2007). Fisher lecture: Dimension Reduction in Regression (with discussion). Statist. Sci. 22 p1-26. 
[3] Cook, R.D., Li, B. and Chiaromonte, F. (2010). Envelope Models for Parsimonious and Efficient Multivariate Linear Regression. Statistica Sinica 20, p927-1010.

[4] Cook, R.D., Li, B. and Chiaromonte, F. (2007). Dimension Reduction without matrix inversion. Biometrika, 94, p569-584.

[5] Halmos, P.R. $(1974,1987)$. Finite-Dimensional Vector Space. Published by Springer-Verlag, New York Inc.

[6] Handbook of Linear Algebra. (2007). Edited by Leslie Hogben,Associate editors, Richard Brualdi, Anne Greenbaum, Roy Mathias. Chapman \& Hall/CRC, Taylor \& Francis Group, Boca Raton, London, New York.

[7] $\mathrm{Mal}^{\wedge} \mathrm{Cev}$, A.I.(1963). Foundations of Linear Algebra. Translated from the Russian by Thomas Craig Brown, Edited by Roberts, J.B. Published by W.H. Freeman and Company, San Francisco and London. 